\title{
A Nanoparticle Delivery of Plasmid Encoding Hepatocyte Growth Factor for Gene Therapy of Silicosis in Mice
}

\author{
Haiying Duan ${ }^{1,2}$, Peng Gao ${ }^{3}$, Xiaochen Cheng ${ }^{2}$, Yuxin $\mathrm{Lu}^{2}$, Chunsheng $\mathrm{Hu}^{4}$, Xuefeng $\mathrm{Zhu}^{1,2}$, Xiaoying Wang ${ }^{1,5}$, Dujuan $\mathrm{Li}^{1,2}$, \\ Fengjun $\mathrm{Xiao}^{2}$, $\mathrm{Li} \mathrm{Du}^{2}$, Yunmei Liu' ${ }^{1}$, Qinglin Zhang ${ }^{2}$ \\ ${ }^{1}$ Institute of Pharmacy \& Pharmacology, University of South China, Hengyang, Hunan, China; ${ }^{2}$ Beijing Institute of Radiation \\ Medicine, Beijing, China; ${ }^{3}$ The Second Affiliated Hospital of Xuzhou Medical University, Xuzhou, Jiangsu, China; ${ }^{4}$ National \\ \& Local Joint Engineering Research Center of Targeted and Innovative Therapeutics, College of Pharmacy \& International \\ Academy of Targeted Therapeutics and Innovation, Chongqing University of Arts and Sciences, Chongqing, China; ${ }^{5}$ College \\ of Chemistry and Environmental Sciences, Hebei University, Baoding, Hebei, China
}

Corresponding authors: $\mathrm{Li} \mathrm{Du}$, Beijing Institute of Radiation Medicine, 27 Taiping Road, Beijing 100850, China; email: duli@nic.bmi.ac.cn; Yunmei Liu, Institute of Pharmacy \& Pharmacology, University of South China, Hengyang, Hunan, China, 421000; email: 2002001791@usc.edu.cn

Received, July 27, 2021; Revised September 21, 2021; Accepted, September 22, 2021; Published, September 25, 2021

\begin{abstract}
Purpose: Silicosis is a serious occupational disease that is characterized by pulmonary infiltrates and fibrosis and is often refractory to current treatments. New therapeutic strategies for silicosis are needed. Hepatocyte growth factor (HGF) is a latent anti-inflammatory and anti-fibrotic growth factor. Methods: We prepared a polyethyleneimine-polyethylene glycol/pHGF/hyaluronic acid (PEG-PEI/pHGF/HA) nanomaterials loaded with plasmid DNA encoding HGF gene to increase its transfection efficiency. The characterization, including DNA entrapment efficiency, morphology, particle size, and zeta-potential of PEG-PEI/pHGF/HA was studied. And a PEG-PEI/pHGF/HA (N/P=30:1) nanoparticle with low toxicity and high transfection efficiency was used in treatment for silicosis in mice. Results: The results showed that the human HGF expression in the lungs of the mice was increased, and the inflammatory cell infiltration and fibrous collagen deposition was significantly reduced. Conclusion: Therefore, PEG-PEI/pHGF/HA nanoparticle warrant further investigation and may be a potential therapeutic strategy for silicosis.
\end{abstract}

\section{INTRODUCTION}

Silicosis, a serious occupational lung disease caused due to continuous inhalation of crystalline silica ( $\mathrm{SiO} 2)$ (particles smaller than $10 \mu \mathrm{m}$ in diameter), is characterized by pulmonary infiltrates and fibrosis (1). At present, patients with silicosis generally receive only supportive treatment, mainly to increase blood oxygen or control infection-oxygenation therapy, antibiotics, and respiratory exercises (2). However, the therapeutic effect of the traditional treatments is not as effective as expected. Lung transplantation may be the only treatment for patients in final stage or advanced phrase (3). Therefore, novel therapeutic approaches that prevent silicosis and delay fibrosis are urgently required.

Hepatocyte growth factor (HGF), a plasminogen-related and mesenchyme-derived pleiotropic growth factor, is secreted by stromal cells and mesenchymal cells (4). Many studies have reported that HGF has several molecular functions including anti-apoptosis (5), anti-inflammatory (6), anti-fibrosis (7), anti-nociceptive (8), etc. In our previous studies, we found that intramuscular injection of a naked plasmid encoding human HGF gene (pHGF) into injured tissue significantly attenuated chronic post-surgical pain induced by skin/muscle incision and retraction. The analgesic effect of HGF was associated with promoting blood flow along with proliferation of satellite cells and inhibiting inflammatory cells recruitment along with collagen accumulation $(9,10)$. In addition, local injection of naked pHGF improved the kidney injury and renal fibrosis induced by gentamicin (7). However, the effect of pHGF on silicosis is still unclear.

Previous studies and our experiments showed that naked plasmid DNA injection was a safe, feasible and economical gene transfer approach (10, 11), but its therapeutic efficacy was limited due to low transfection and transgene expression in lungs by inhalation. In order to address this issue, various gene carriers, such as polyethyleneimine (PEI), polyamidoamine (PAM), along with arginine-rich 
peptides, were developed to improve the transfection efficiency of therapeutic plasmid DNA into the lungs (12). PEI is widely used in gene delivery because of its proton sponge effect and excellent gene expression (13). However, the toxicity of PEI-based vectors poses a serious challenge for developing safe and successful gene therapies. Various strategies, including conjugation of polyethylene glycol (PEG), modification with folic acid (FA), arginylglycylaspartic acid (RGD) and isoleucinelysine-valinealanine-valine (IKVAV) peptides, have been used to overcome the limitations of PEI (14). Masaki et al. have transferred hHGF gene into the lung by MAA-PEI, resulting in reduced inflammation and fibrosis after bleomycin injury (15). Studies have found that PEG-modified PEI could reduce its cytotoxicity. However, PEG modification could neutralize the positive charge of PEI and change its configuration, leading to the reduction of the transfection efficiency (16). To improve transfection efficiency and targeting, hyaluronic acid (HA) was introduced to modify PEG-PEI. HA is a linear macromolecule of mucopolysaccharide, consisting of alternately linked glucuronic acid and $\mathrm{N}$-acetylglucosamine (17). HA is highly viscous, non-immunogenic, biocompatible, and degradable (18). HA and polycation coupling have significantly increased serum stability and receptor-mediated endocytosis (19). HA modified PEI demonstrated low cytotoxicity but high gene transfection efficiency (20).

In the current study, a combination with pHGF and HA modified PEG-PEI nanomaterials (PEG$\mathrm{PEI} / \mathrm{pHGF} / \mathrm{HA}$ ) was evaluated in mouse silicosis model. The PEG-PEI/pHGF/HA gene therapy was low toxicity and high transfection efficiency in lungs of mice. After treatment with PEG-PEI/pHGF/HA, the HGF expression in the lungs of the mice was increased, and the deposition of collagen was significantly reduced. The results suggested that the PEG-PEI/pHGF/HA gene therapy has a potential to treat the refractory silicosis.

\section{MATERIALS AND METHODS}

\section{Materials}

Methoxypoly (ethylene glycol) succinimidyl propionate (m-PEG-SPA, Mw=5 $\mathrm{kDa}$ ) was obtained from Chemgen Pharma (Beijing, China); HA $(\mathrm{Mw}=10 \mathrm{kDa})$ was obtained from Bloomage Freda Biopharm Co., Ltd (Shandong, China); b-PEI $(\mathrm{Mw}=25 \mathrm{kDa})$ and silica (Mw: $60.08 \mathrm{~g} / \mathrm{mol}$, monodisperse, non-porous, $3.0 \mu \mathrm{m}$ ) were purchased from Sigma-Aldrich (St. Louis, MO, USA); CCK-8 was obtained from Bimake.cn. Male C57BL/6 mice $(20 \pm 2$ g) were obtained from SPF (Beijing) Biotechnology Co., Ltd. The plasmid carried human HGF gene(pHGF) was prepared in our laboratory. The iQuant high sensitivity dsDNA quantitation kit was purchased from GeneCopoeia Expressway to Discovery (USA). HGF antibody (Catalog No. DF2838290) was purchased from Affinity Biosciences LTD.

\section{Synthesis of PEG-PEI}

PEG-PEI was synthesized using b-PEI as a terminal amino donor and functional m-PEG-SPA as an acetylation reagent (21). $73.5 \mathrm{mg}$ b-PEI and $2.5 \mathrm{~mL}$ $\mathrm{ddH} 2 \mathrm{O}$ were added to a flask and stirred at room temperature (RT) to ensure that b-PEI is fully dissolved in water. $300.3 \mathrm{mg}$ of m-PEG-SPA was dissolved in $2.5 \mathrm{~mL} \mathrm{ddH2O}$, the resulting solution was added dropwise to the flask containing b-PEI solution with stirring at RT. The molar ratio of $\mathrm{m}$ PEG-SPA and b-PEI was 20:1. The solution obtained was added to an ultrafiltration tube (molecular weight cut-off, MWCO: $30 \mathrm{kDa}$ ) followed by centrifugation at $6000 \mathrm{rpm}$ for $20 \mathrm{~min}$. The solution was centrifuged twice after adding three times $\mathrm{ddH} 2 \mathrm{O}$. Finally, the solution was lyophilized in a freeze dryer (LGJ-25C Freeze Dryer, Beijing Sihuan Scientific Instrument Factory Co., Ltd) at $-45^{\circ} \mathrm{C}$ for $6 \mathrm{~h}$ to obtain the PEG-PEI powder. The chemical structure was confirmed using $1 \mathrm{H}$ NMR (in D2O, $400 \mathrm{MHz})$ and Infra-Red detection (KBr).

\section{Preparation of pHGF-loaded PEG-PEI (HA) nanoparticles}

The N/P ratio of PEG-PEI/pHGF/HA is the ratio of moles of amine groups of PEG-PEI to the moles of phosphate groups of pHGF. PEG-PEI/pHGF complexes were allowed to self-assemble in ddH2O by mixing the $\mathrm{pHGF}$ solution $(1 \mathrm{mg} / \mathrm{mL})$ with PEGPEI solution $(1 \mathrm{mg} / \mathrm{mL})$ at a certain N/P ratio. The amount of pHGF was kept constant, and the mixture was allowed to stand for $20 \mathrm{~min}$ at room temperature before use. PEG-PEI/pHGF/HA complexes were induced to self-assemble in ddH2O by mixing PEG$\mathrm{PEI} / \mathrm{pHGF}$ with $\mathrm{HA}$ solution $(1 \mathrm{mg} / \mathrm{mL})$ and allowing to stand for $30 \mathrm{~min}$ at room temperature. The mass ratio of HA to PEG-PEI was 1.

\section{Characterization of PEG-PEI/pHGF/HA complexes}

The condensation capability of PEG-PEI/pHGF/HA was evaluated using agarose gel electrophoresis. 
PEG-PEI/pHGF/HA complexes were prepared at various N/P ratios and electrophoresed on a $0.8 \%$ (w/v) agarose gel in Tris/Borate/EDTA (TBE) buffer. The electrophoresis results were analyzed using an ultraviolet gel imager (Beijing Saizhi Venture Technology Co. Ltd). Naked dsDNA in solution was quantified by measuring the absorbance at $485 \mathrm{~nm}$ excitation wavelength (Ex) and $530 \mathrm{~nm}$ emission wavelength (Em) using a Qubit4 fluorometer (Quantifiers Semer Technology Co., Ltd, China).

The entrapment efficiency was calculated as follows: Entrapment efficiency $(\%)=(\mathrm{Ft}-$ $\mathrm{Ff}) / \mathrm{Ft} \times 100 \%$, (Ft - total DNA, Ff - free DNA). The particle size and zeta-potential values of PEG$\mathrm{PEI} / \mathrm{pHGF} / \mathrm{HA}$ at various N/P ratios were determined using a particle size analyzer (Malvern Panalytical, UK). The particle morphology was observed under a transmission electron microscope (Hitachi, H-7650, Japan).

\section{HGF Expression in Cell culture}

A549 cells were cultured in Dulbecco's Modified Eagle Medium (DMEM) medium with $10 \%$ fetal bovine serum (FBS) in an atmosphere of $5 \% \mathrm{CO} 2$ inside a humidified cell incubator. The A549 cells were seeded in 24-well flat-bottom plates at the density of $1 \times 105$ cells/well. The cells were grown overnight up to $70-80 \%$ confluence. The various N/P ratios of PEG-PEI/pHGF/HA were diluted with serum-free medium and added to the wells. The amount of pHGF was fixed at $500 \mathrm{ng} /$ well. After $6 \mathrm{~h}$ incubation, the media were replaced with fresh media containing $10 \%$ FBS.

The expression of HGF in A549 cells was determined using an ELISA kit (Multi Sciences, China) at $48 \mathrm{~h}$ after transfection.

\section{Cytotoxicity evaluation}

The cytotoxicity of PEG-PEI/pHGF/HA were evaluated using CCK-8 assay. A549 cells were seeded in 96-well plates at the density of 5000 cells/well. The cells were treated with PEG$\mathrm{PEI} / \mathrm{pHGF} / \mathrm{HA}$ (sample) or pHGF (control) for $24 \mathrm{~h}$. Then $10 \mu \mathrm{L}$ of $5 \mathrm{mg} / \mathrm{mL}$ CCK-8 solution was added to the cells, and the plates were incubated for $4 \mathrm{~h}$ at $37^{\circ} \mathrm{C}$. The absorbance values were measured at 450 nm using the Multiskan MK3 enzyme-labeled instrument (Thermo, US). Cell viability was calculated based on the absorbance values of the sample and the control group.

\section{Administration of silica and PEG-PEI/pHGF/HA through the mouse trachea}

Before silica administration, silica was baked at $200^{\circ} \mathrm{C}$ for $4 \mathrm{~h}$ to remove endotoxins. The silica was then dissolved in PBS to prepare a $50 \mathrm{~g} / \mathrm{L}$ suspension. The mice (12 $\mathrm{h}$ fasted) were anesthetized with $1 \%$ sodium pentobarbital through intraperitoneal injection. After finding the trachea through a laryngoscope, a lung quantitative nebulizer (Beijing Huironghe Technology Co., Ltd.) was inserted into the trachea and aerosolized $100 \mu \mathrm{L}$ suspension of silica into the lung.

120 male C57BL/6 mice $(20 \pm 2 \mathrm{~g})$ mice were randomly divided into four groups: saline group, silicosis model group (model group), pHGF group, and PEG-PEI/pHGF/HA group ( $\mathrm{n}=30$ in each group). Mice in the saline group were administrated with saline through the trachea. The mice in model, pHGF, and PEG-PEI/pHGF/HA groups were administered with silica through the trachea. On day 1 and day 7 post-silica-administration, the mice in the model, pHGF, and PEG-PEI/pHGF/HA groups, were administered with saline, pHGF, and PEG$\mathrm{PEI} / \mathrm{pHGF} / \mathrm{HA}$, respectively through the trachea.

All animal experiments complied with the ARRIVE guidelines and be carried out in accordance with the National Institutes of Health guide for the care and use of Laboratory animals (NIH Publications No. 8023, revised 1978).

\section{Pulmonary function detection}

On the 7th, 14th and 28th day after modeling, 5 mice/group were tested for lung function detection. After the mice were calm, the pulmonary function of the mice was detected with non-invasive pulmonary function test (Shanghai TOW Intelligent Technology Co., Ltd.). Then the respiratory function indexes of the mice were measured, including tidal volume (TV), peak expiratory flow (PEF), peak inspiratory flow (PIF) and expiratory flow rate at 50\% exhaled volume (EF50).

\section{Hematoxylin-Eosin and Masson's trichrome staining}

The left lungs of the mice were perfused with $10 \%$ neutral buffered formalin, fixed overnight, dehydrated, and embedded in paraffin. Thick sections $(5 \mu \mathrm{m})$ were cut and mounted on the slides, followed by deparaffinization. The sections were stained with hematoxylin and eosin to determine pulmonary pathology and with Masson staining to visualize collagen deposition. 


\section{Immunohistochemistry}

The tissue sections were deparaffinized and treated with $3 \% \mathrm{H} 2 \mathrm{O} 2$ in phosphate-buffered saline for 15 min, blocked with $5 \%$ goat serum, and incubated with primary antibody at $4^{\circ} \mathrm{C}$ overnight. After three washes, the sections were incubated with horseradish peroxidase-conjugated secondary antibody for 20 $\min$ at room temperature. The nuclei were then stained with DAB solution and visualized under a fluorescent microscope (DM750, Leica, Germany).

\section{Detection of hydroxyproline}

Hydroxyproline (HYP) assay was conducted using an HYP test kit (Nanjing Jiancheng Bioengineering Institute (Nanjing, China)). The assay was carried out according to the manufacturer's instructions.

\section{Statistics}

Data are presented as mean $\pm \mathrm{SD}$, and each measurement was performed in triplicate. Differences were analyzed by a one-way ANOVA followed by the Fisher's least significant difference (LSD) test to determine differences between groups for normally distributed continuous variables or by Kruskal-Wallis H test followed by Mann-Whitney $\mathrm{U}$ test to determine differences between groups for non-normally distributed continuous variables. $\mathrm{P}<0.05$ was considered to be statistically significant.

\section{RESULTS}

\section{Structural identification and characterization of the compounds}

Structure of PEG-PEI copolymer. Figure 1 showed the synthetic route of PEG-PEI. When PEG and PEI were reacted at a molar ratio of 20:1, the product mass was $276.3 \mathrm{mg}$, and the yield of the compound was $94.9 \%$. PEG-PEI was characterized by $1 \mathrm{H}$ NMR and IR. The absorption around $1740 \mathrm{~cm}-1$ ( $\mathrm{s}$, v easter $-\mathrm{C}=\mathrm{O}$ ) in the infrared spectrum was weakened, and the absorption at $3421 \mathrm{~cm}-1$ (s, v amide $-\mathrm{NH})$ and $1647 \mathrm{~cm}-1 \quad(\mathrm{~s}, \quad \mathrm{v}$ amide $-\mathrm{C}=\mathrm{O})$ appeared, corresponding to the amide linkage. Based on the peak area integration ratio of the polyethylene glycol and the polyethyleneimine unit $(3.09: 1)$, their grafting ratio was $15.8: 1$. The molecular weight of PEG-PEI was about $100 \mathrm{kDa}$.

The particle size and zeta-potentialc of PEG$\boldsymbol{P E I / p H G F / H A}$. As shown in Figure 2A, PEG$\mathrm{PEI} / \mathrm{pHGF} / \mathrm{HA}$ complexes were induced to assemble in ddH2O by mixing PEG-PEI/pHGF with HA solution. The PEG-PEI/pHGF and PEG-
$\mathrm{PEI} / \mathrm{pHGF} / \mathrm{HA}$ complexes were formed by mixing with different N/P ratios. The morphology of PEG$\mathrm{PEI} / \mathrm{pHGF} / \mathrm{HA}$ was examined using transmission electron microscopy (TEM) (Figure 2B). The particle size of the PEG-PEI/pHGF/HA gradually reduced from $185 \mathrm{~nm}$ to $135 \mathrm{~nm}$ when the N/P ratio increased form 5:1 to 30:1, which became stable and was $135 \mathrm{~nm}$ as the N/P ratio reached 20:1. The PEG$\mathrm{PEI} / \mathrm{pHGF} / \mathrm{HA}$ had a larger particle size compared to the PEG-PEI/pHGF (Figure 2C). The zeta-potential of PEG-PEI/pHGF complex was positive charge, however, the PEG-PEI/pHGF/HA complex was negative charge. Furthermore, the zeta-potential of PEG-PEI/pHGF/HA complex increased with the increase of N/P ratio (Figure 2D). In addition, the gel electrophoresis showed that PEG-PEI/pHGF/HA complex was able to completely wrap pHGF above N/P ratio at 4:1. (Table 1, Figure 2E).

Table 1. Encapsulation rates $(\%)$ of various carriers at $\mathrm{N} / \mathrm{P}=1-6$

\begin{tabular}{lllllll}
\hline $\begin{array}{l}\text { Carrier and } \\
\text { NP ratio }\end{array}$ & $\mathbf{N} / \mathbf{P}=\mathbf{1}$ & $\mathbf{N} / \mathbf{P}=\mathbf{2}$ & $\mathbf{N} / \mathbf{P}=\mathbf{3}$ & $\mathbf{N} / \mathbf{P}=\mathbf{4}$ & $\mathbf{N} / \mathbf{P}=\mathbf{5}$ & $\mathbf{N} / \mathbf{P}=\mathbf{6}$ \\
PEI & $47.7 \%$ & $93.1 \%$ & $99.6 \%$ & $99.8 \%$ & $99.8 \%$ & $99.8 \%$ \\
PEG-PEI & $50.1 \%$ & $87.7 \%$ & $87.8 \%$ & $96.9 \%$ & $96.9 \%$ & $99.8 \%$ \\
PEG-PEI/HA & $49.6 \%$ & $88.6 \%$ & $88.6 \%$ & $98.8 \%$ & $99.8 \%$ & $99.8 \%$ \\
\hline
\end{tabular}

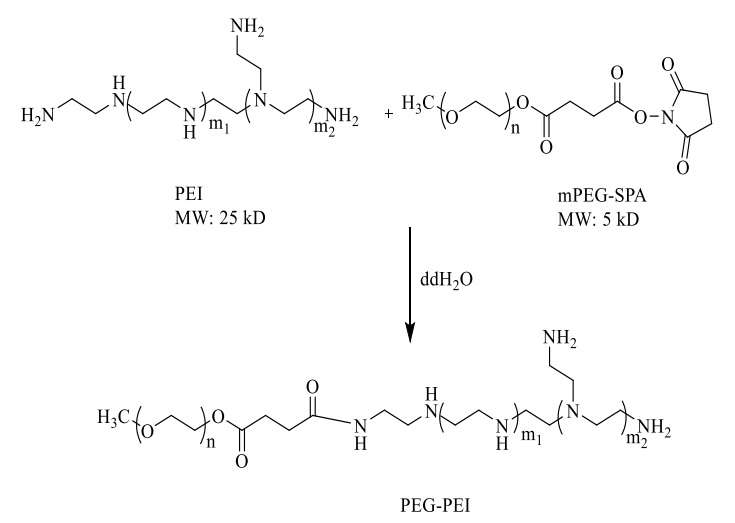

Figure 1. The synthesis route of PEG-PEI.

\section{The bioactivity of PEG-PEI/pHGF/HA in vitro}

Transfection efficiency and cytotoxicity of PEG$P E I / p H G F / H A$ in vitro. The transfection efficiency of PEG-PEI/pHGF/HA or PEG-PEI/pHGF complex was conducted by detecting the human HGF protein in A549 cells with ELISA. As shown in Figure3A, the PEG-PEI/pHGF/HA complex increased the levels of HGF from 211 to $332 \mathrm{pg} / \mathrm{ml}$ as N/P ratio increased form 10:1 to 30:1, which were also similar to that of the PEG-PEI/pHGF complex. When the $\mathrm{N} / \mathrm{P}$ ratio was $30: 1$, the transfection efficiency of the 


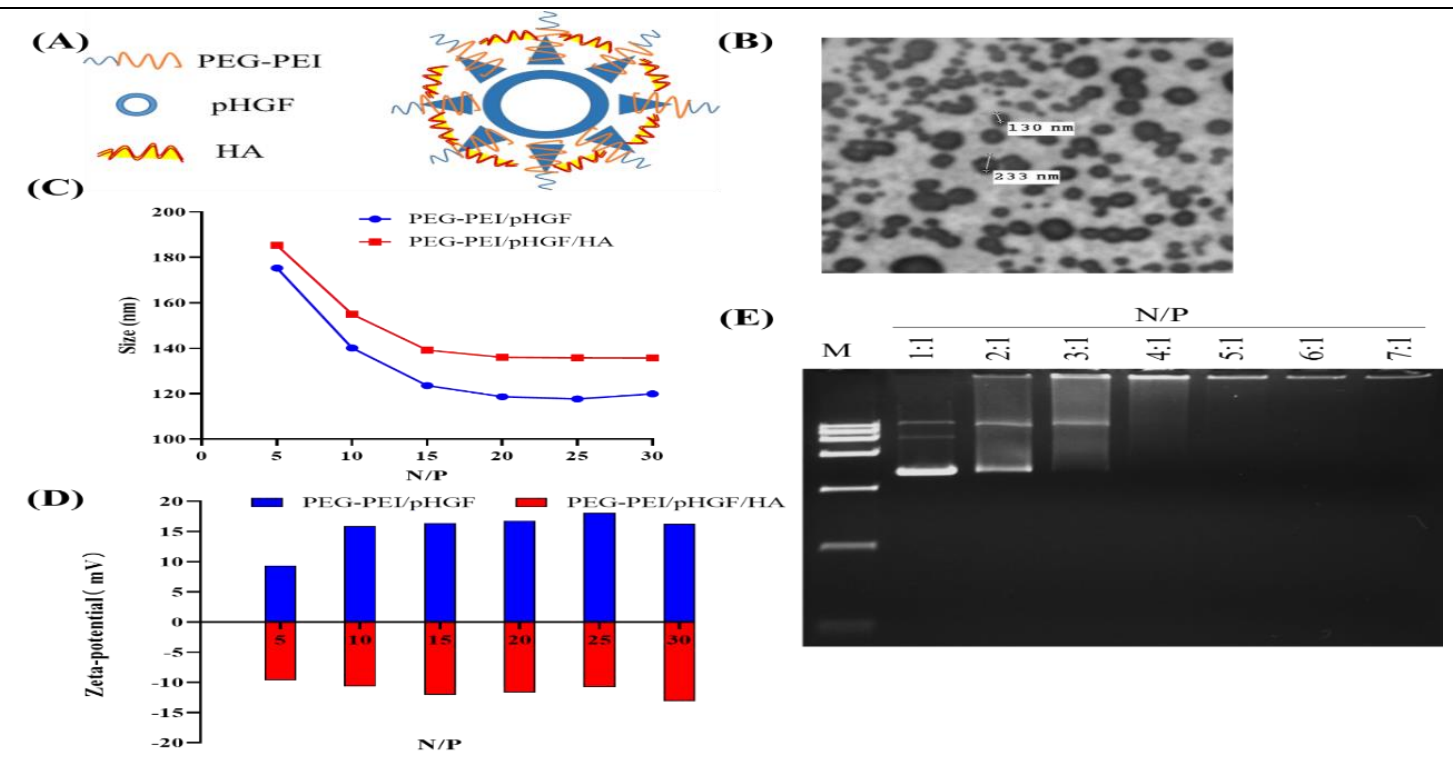

Figure 2. The particle size, zeta-potential and entrapment efficency of PEG-PEI/pHGF/HA. (A) A schematic of assemble for PEG-PEI/pHGF/HA. (B) Transmission electron microscopy (TEM) image of PEG-PEI/pHGF/HA with N/P ratio at 30:1 $(12000 \times)$. (C) The particle size of PEG-PEI/pHGF/HA and PEG-PEI/pHGF with different N/P. (D) The zeta-potential of PEG-PEI/pHGF/HA with different N/P. (E) Entrapment of PEG-PEI/pHGF /HA with different N/P measured by gel electrophoresis. M: Ladder, N/P ratios ranging from 1:1 to 7:1.

A549 cells after treatment with PEG-PEI/pHGF/HA at $\mathrm{N} / \mathrm{P}=10: 1,20: 1,30: 1$ and 40:1. When N/P ratio was $30: 1$ or $40: 1$, the cell survival rate was over $85 \%$. PEG-PEI/pHGF/HA complex was significantly higher than that of the pHGF group $(\mathrm{P}<0.01)$. The cytotoxicity results showed that there was no significant difference in the cell survival rates of Compared to PEG-PEI/pHGF/HA complex, the PEG-PEI/pHGF complex exerted a greater cytotoxic effect (Figure 3B). The results demonstrate that PEG-PEI/pHGF/HA has favorable transfection efficiency and lower cytotoxicity.

\section{The therapeutic effect on silicosis in mice of PEG- PEI/pHGF/HA in vivo}

HGF expression and changes in lung function on silicosis. To investigate whether PEG$\mathrm{PEI} / \mathrm{pHGF} / \mathrm{HA}$ has therapeutic effect on silicosis in vivo, a mouse model of silicosis was established and then intratracheal administration of PEG$\mathrm{PEI} / \mathrm{pHGF} / \mathrm{HA}$ was performed at 1 and 7 days after silica administration. As shown in Figure 4B, intratracheal administration of silica significantly induced infiltration of inflammatory cells and alveolar septal thickening in lung at day 7 by pathological examination. After intratracheal administration of PEG-PEI/pHGF/HA, the expression of HGF protein in pulmonary epithelial cell was increased in the pHGF group and PEG$\mathrm{PEI} / \mathrm{pHGF} / \mathrm{HA}$ group compared to that in saline group (Figure 4C) at day 14, indicating that pHGF can be delivered into the lung by the nanoparticles and HGF can be expressed in epithelial cells.

The respiratory function indexes, including PIF, PEF, TV and EF50, were decreased in the model group at days 7,14 and 28, while these indexes were increased after treatment with PEG-PEI/pHGF/HA at days 7, 14 and 28 as shown in Figure 4D. Treatment with PEI/pHGF/HA resulted in increased PIF, PEF, TV and EF50. These results suggested that intratracheal administration of PEG-PEI/pHGF/HA improved pulmonary function in mice with silicosis.

Pathological improvement after treatment with PEG-PEI/pHGF/HA. As shown in Figure 5A, there were massive silica crystals in the lung of mice in model group, $\mathrm{pHGF}$ group and PEG-PEI/pHGF/HA group. The inflammatory cell infiltration and alveolar septal thickening were significantly alleviated in the PEG-PEI/pHGF/HA group compared with that in the model group. 

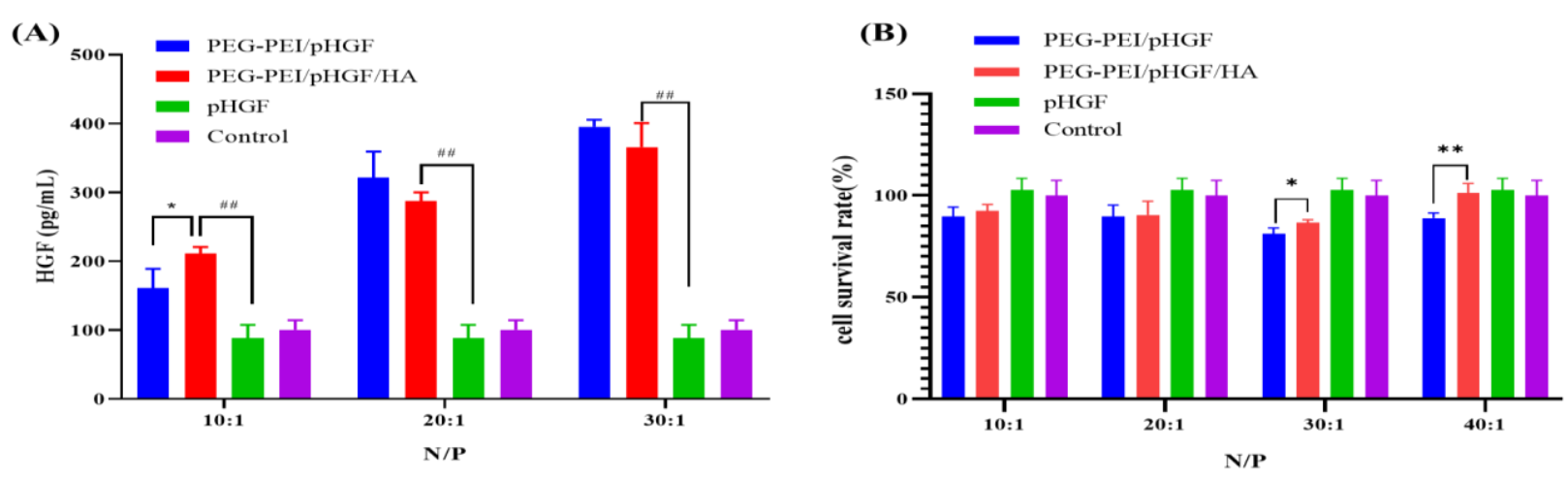

Figure 3. Transfection efficiency and cytotoxicity of PEG-PEI/pHGF/HA. (A) Expression of HGF in A549 cells detected by ELISA. PEG-PEI/pHGF/HA at different N/P $(10,20,30)$, Blank: culture medium. $\left({ }^{*} P<0.05\right.$ vs PEGPEI/pHGF, \#\#P<0.01 vs pHGF). (B) Cell survival rates of A549 cells with different treatment $(* P<0.05$, **

$P<0.01$ vs $P E G-P E I / p H G F)$.

The content of HYP in PEG-PEI/pHGF/HA group were reduced significantly as shown in Figure $5 \mathrm{~B}$, indicating that the content of collagen in the lungs decreased after PEG-PEI/pHGF/HA administration. Consistent with the HYP results, collagen accumulation using Masson staining was significantly reduced in PEG-PEI/pHGF/HA group compared with the model group (Figure 5C).

\section{DISCUSSION}

HGF, as a pleiotropic cytokine, plays an anti-fibrosis role by promoting the repair of epithelial and endothelial cells, enhancing collagenase activity, and inhibiting myofibroblast accumulation (22) by binding specifically to the tyrosine kinase membrane receptor c-met (23). HGF prevents the death of respiratory epithelial cells and promotes the orderly regeneration of the peripheral respiratory tract. HGF also protects against lung injury induced by bleomycin (24). Besides, HGF is a latent antiinflammatory and anti-fibrotic growth factor (25). Therefore, HGF is a potential anti-pulmonary fibrosis candidate gene drug for treating silicosis. In this study, we prepared plasmid nanoparticles carrying human HGF gene, PEG-PEI/pHGF/HA, with high transfection efficiency and low cytotoxicity. Human $\mathrm{HGF}$ proteins was greatly increased ranging from day 7 to day 28 after intratracheal administration of PEG-PEI/pHGF/HA. In addition, the PEG-PEI/pHGF/HA improved the pulmonary function and inhibited inflammatory cell infiltration and fibrous collagen deposition in mice with silicosis. These results suggest that PEG$\mathrm{PEI} / \mathrm{pHGF} / \mathrm{HA}$ may provide a potential approach for controlling the silicosis.
Naked therapeutic genes have to overcome various physical barriers, including endo-lysosome entrapment, cytoplasmic isolation, and nuclear rejection of DNA during transportation. The efficiency is further reduced due to nuclease degradation (26). Hence, one of the main challenges of the clinical application of gene therapy is to develop a safe and efficient gene delivery carrier (27). In current study, we introduce HA into the structural design of plasmid DNA transport in vivo, because HA-structured nanoparticles can prolong the mean residence time (MRT) of drugs in the lungs (28). After chemical synthesis of PEG$\mathrm{PEI} / \mathrm{pHGF} / \mathrm{HA}$, the physical and chemical properties of the nanoparticles were tested. As the N/P ratio increased ranging from 20:1 to 30:1, the nanoparticle size was stabilized at $135 \mathrm{~nm}$. Furthermore, the addition of HA resulted in a negative potential. Several studies suggested that particles under $200 \mathrm{~nm}$ were not recognized by macrophages, and hollow particles with low density and large size were better deposited than small solid particles with high density $(29,30,31)$. HA affected the membrane potential through electrostatic interactions with lipids, resulting in lipid leakage (32). Our results showed that the transfection efficiency of PEG$\mathrm{PEI} / \mathrm{pHGF} / \mathrm{HA}$ nanoparticle increased with the increase of N/P in vitro, indicating that the size and potential of the synthesized nanoparticles are suitable for delivering pHGF into cells. Additionally, the cytotoxic of $\mathrm{PEG}-\mathrm{PEI} / \mathrm{pHGF} / \mathrm{HA}(\mathrm{N} / \mathrm{P}=30: 1)$ was relatively low in the cell culture, and the mice did not exhibit any toxicity symbols after administrated with PEG-PEI/pHGF/HA (N/P=30:1).

The progression of silicosis is a multi-factorial process. Crystalline silicon induces persistent pneumonia and excessive production of extracellular 
(A)

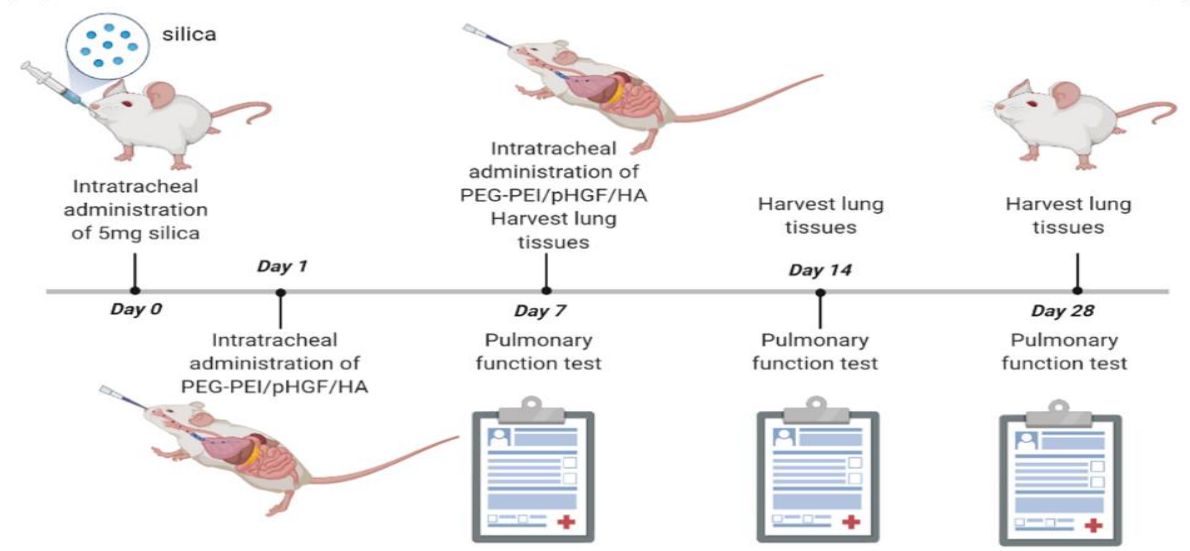

(B)

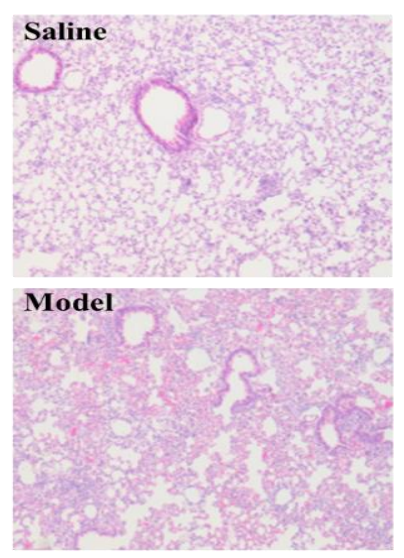

(C)

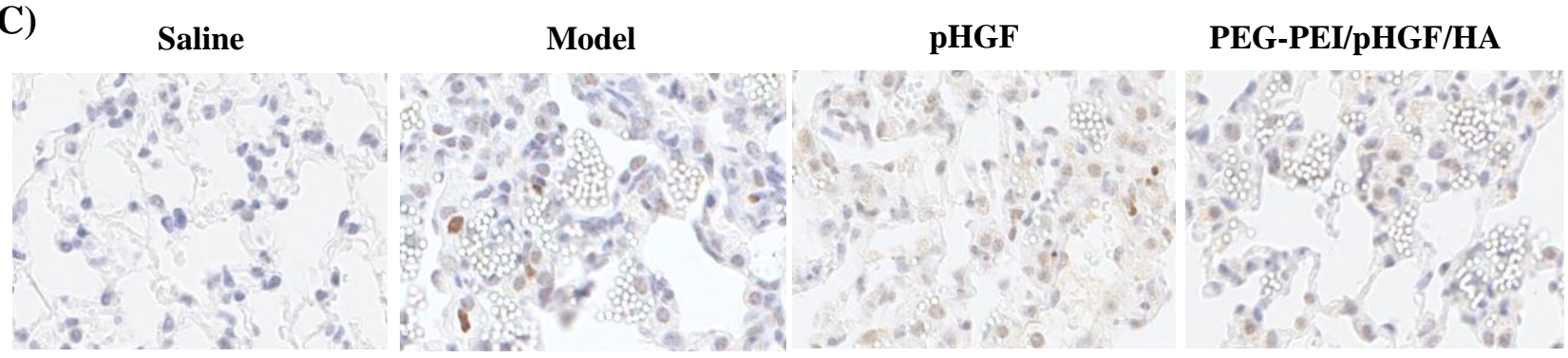

(D)

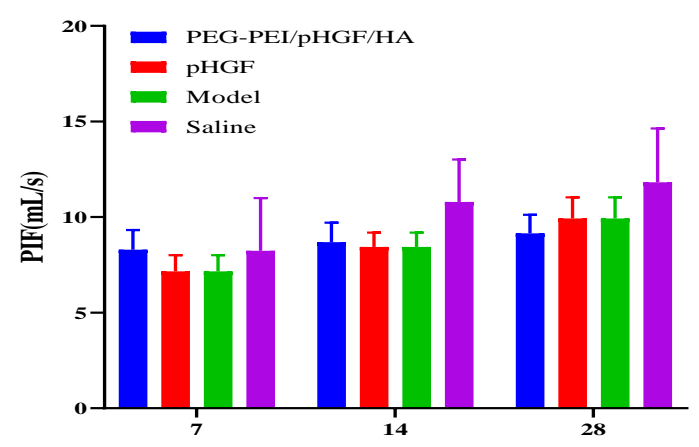

Day after treatment

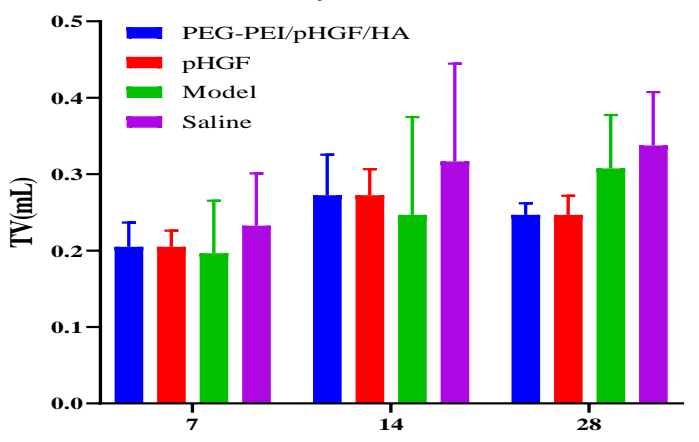

Day after treatment

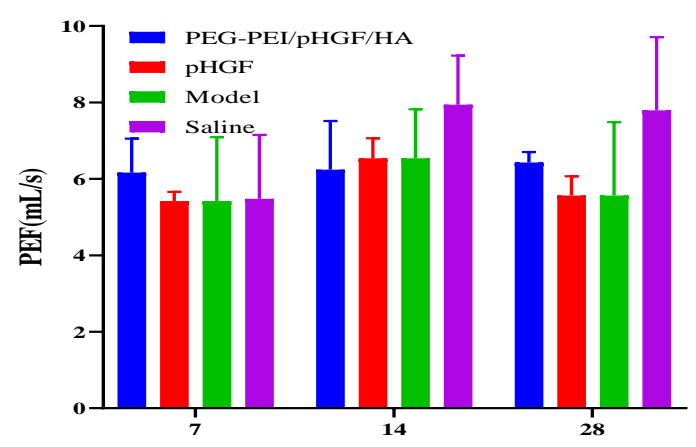

Day after treatment

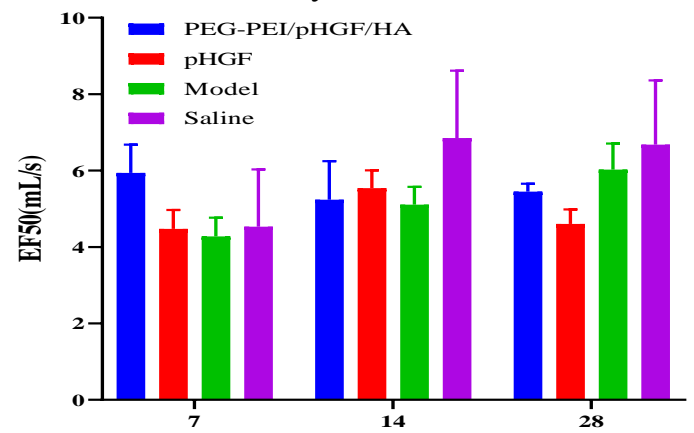

Day after treatment

Figure 4. The HGF expression and therapeutic effects on silicosis after intratacheal administration of PEG-PEI/pHGF/HA. (A) Schematic of mouse silicosis model establishment and PEG-PEI/pHGF/HA administration. (B) Hematoxylin and eosin staining of the lung tissues of mice on day 7 after modeling. (C) HGF expression in the lungs of mice in various groups at day 14 detected by immunohistochemistry staining. (D) Respiratory function analysis including PIF, PEF, TV and EF50 was measured on days 7, 14 and 28 using a lung function detector. 
(A)

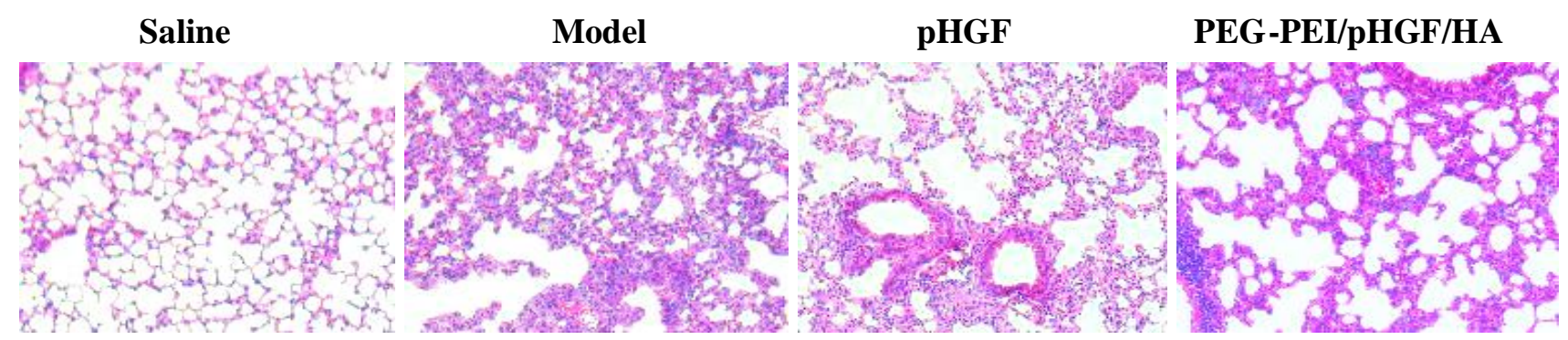

(B)

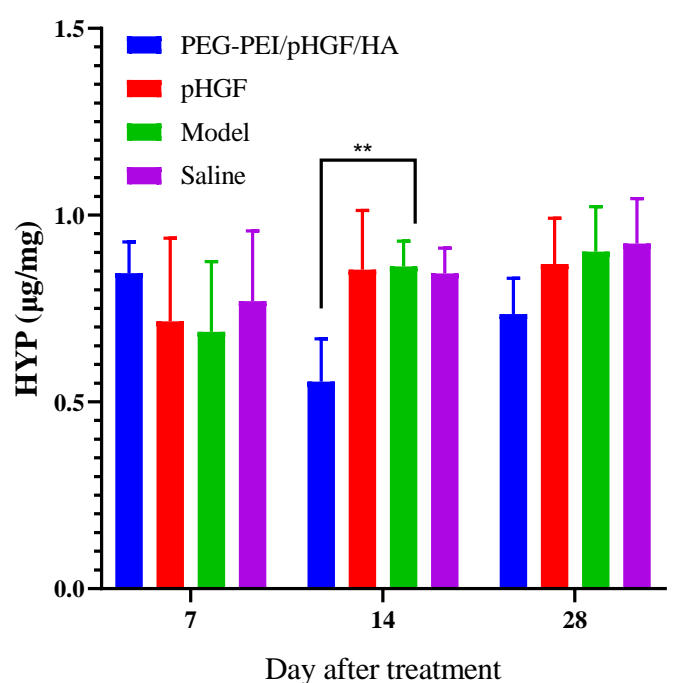

(C)

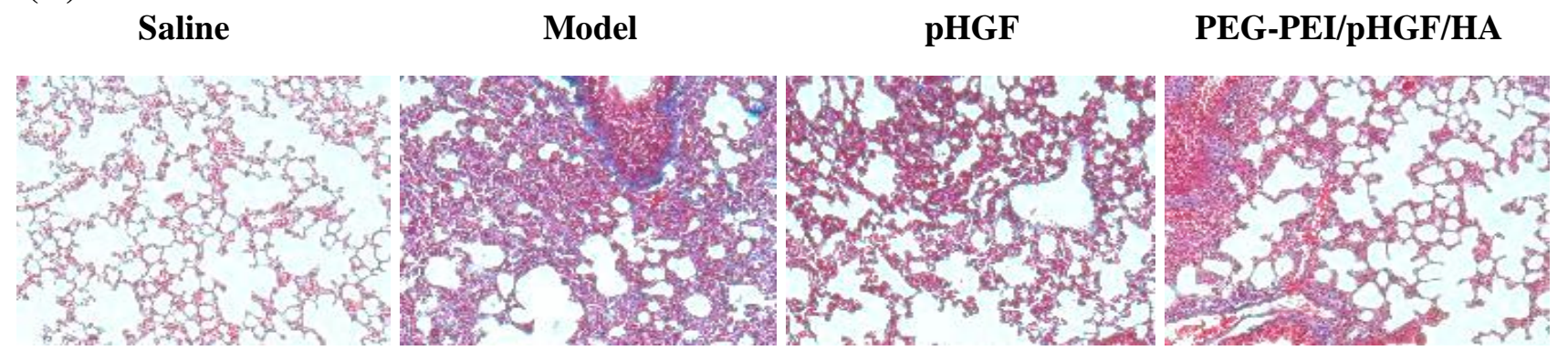

Figure 5. Effect of PEG-PEI/pHGF/HA on the mouse silicosis. (A) Hematoxylin and eosin staining of the lung at day 28 in different groups. (B)The HYP content in the lung tissues in different groups ( $* * P<0.01$, vs. Model). (C) Masson's staining of the lung at day 28 in different groups.

matrix (ECM) (33). Pulmonary fibrosis induced by crystalline silicon begins with a prominent acute inflammatory response caused by recruitment and accumulation of inflammatory cells (macrophages and lymphocytes) and elevation of the levels of proinflammatory and pro-fibrotic cytokines and chemokines (34). During fibrosis, innate and adaptive immune responses are activated, promoting the expression of fibrotic growth factors, cytokines, and chemokines. These manifestations lead to the progression of fibrosis, which is an essential part of the pathogenesis of silicosis $(35,36,37,38)$. Our results showed that inflammatory cell infiltration in the lung was serious at early stage, and then collagen fiber deposited apparently. After PEG$\mathrm{PEI} / \mathrm{pHGF} / \mathrm{HA}$ treatment, the expression of HGF in 
the lungs was increased, and the inflammatory cell infiltration and collagen fiber deposition in the lung reduced. The content of HYP in the lungs of the PEG-PEI/pHGF/HA group was significantly lower than that in the silicosis model group at 14 days after modeling. These results suggest that PEG$\mathrm{PEI} / \mathrm{pHGF} / \mathrm{HA}$ nanoparticles administration could deliver pHGF into the lungs and increase the expression of HGF in the lungs.

\section{CONCLUSION}

In summary, we constructed the PEG-PEI/pHGF/HA nanoparticles carrying HGF gene through chemical synthesis. pHGF was delivered into the lung effectively via PEG-PEI(HA) and resulted in reduced inflammation and collagen deposition in the lungs of silicosis mice. So, the gene therapy with PEG$\mathrm{PE} / \mathrm{pHGF} / \mathrm{HA}$ nanoparticles is a promising strategy for the treatment of silicosis, and provides research foundation and novel ideas for the treatment of silicosis.

ACKNOWLEDGEMENT. The work to produce this review did not receive any specific grant.

CONFLICTS OF INTEREST. None.

AUTHOR CONTRIBUTIONS. Li Du, Peng Gao, Yunmei Liu and Qinglin Zhang designed the study. Haiying Duan, Xiaochen Cheng, Yuxin Lu, Fengjun Xiao, Xuefeng Zhu, Xiaoying Wang, and Dujuan Li performed the experiments and analyzed data. Haiying Duan and Chunsheng $\mathrm{Hu}$ wrote the manuscript. All authors approved the final version of the manuscript.

\section{REFERENCES}

1. Fernandez Alvarez R, Martinez Gonzalez C, Quero Martinez A, et al. Guidelines for the diagnosis and monitoring of silicosis[J]. Arch Bronconeumol, 2015, 51(2): 86-93. https://doi.org/10.1016/j.arbres.2014.07.010

2. Tan S, Chen S. Macrophage Autophagy and Silicosis: Current Perspective and Latest Insights[J]. Int J Mol Sci, 2021, 22(1): 453. https://doi.org/10.3390/ijms22010453

3. Singer J P, Chen H, Phelan T, et al. Survival following lung transplantation for silicosis and other occupational lung diseases[J]. Occup Med (Lond), 2012, 62(2): 134-137. https://doi.org/10.1093/occmed/kqr171
4. Wang $\mathrm{L}$ S, Wang $\mathrm{H}$, Zhang $\mathrm{Q} \mathrm{L}$, et al. Hepatocyte Growth Factor Gene Therapy for Ischemic Diseases[J]. Hum Gene Ther, 2018, 29(4): 413-423. https://doi.org/10.1089/hum.2017.217

5. Motoi S, Toyoda H, Obara T, et al. AntiApoptotic Effects of Recombinant Human Hepatocyte Growth Factor on Hepatocytes Were Associated with Intrahepatic Hemorrhage Suppression Indicated by the Preservation of Prothrombin Time[J]. Int $\mathbf{J}$ Mol Sci, 2019, 20(8): 1821. https://doi.org/10.3390/ijms20081821

6. Rong S L, Wang X L, Wang Y C, et al. Antiinflammatory activities of hepatocyte growth factor in post-ischemic heart failure[J]. Acta Pharmacol Sin, 2018, 39(10): 1613-1621. https://doi.org/10.1038/aps.2018.14

7. Chen $\mathrm{X}$, Chen $\mathrm{Z}$, Wang $\mathrm{H}$, et al. Plasmid pUDK-HGF encoding human hepatocyte growth factor gene attenuates gentamicininduced kidney injury in rats[J]. Exp Toxicol Pathol, 2013, 65(5): 541-547. https://doi: 10.1016/j.etp.2012.03.003

8. Hu C, Lu Y, Cheng X, et al. Gene therapy for neuropathic pain induced by spared nerve injury with naked plasmid encoding hepatocyte growth factor[J]. J Gene Med, 2017, 19(12). https://doi: 10.1002/jgm.2994

9. $\mathrm{Hu} \mathrm{C}, \mathrm{Lu} \mathrm{Y}, \mathrm{Chen} \mathrm{X}$, et al. Gene transfer of a naked plasmid (pUDK-HGF) encoding human hepatocyte growth factor attenuates skin/muscle incision and retraction-induced chronic post-surgical pain in rats[J]. Eur $\mathrm{J}$ Pain, 2018, 22(5): 961-972. https://doi: 10.1002/ejp.1182.

10. Cui S, Guo L, Li X, et al. Clinical Safety and Preliminary Efficacy of Plasmid pUDK-HGF Expressing Human Hepatocyte Growth Factor (HGF) in Patients with Critical Limb Ischemia[J]. Eur J Vasc Endovasc Surg, 2015, 50(4): 494-501. https://doi: 10.1016/j.ejvs.2015.05.007

11. Elizaga M L, Li S S, Kochar N K, et al. Safety and tolerability of HIV-1 multiantigen pDNA vaccine given with IL-12 plasmid DNA via electroporation, boosted with a recombinant vesicular stomatitis virus HIV Gag vaccine in healthy volunteers in a randomized, controlled clinical trial[J]. PLoS One, 2018, 13(9): e0202753.

10.1371/journal.pone.0202753 
12. Piao C, Zhuang C, Choi M, et al. A RAGEantagonist peptide potentiates polymeric micelle-mediated intracellular delivery of plasmid DNA for acute lung injury gene therapy[J]. Nanoscale, 2020, 12(25): 1360613617. https://doi.org/10.1039/d0nr01367f

13. Chen G G, Mao M, Qiu L Z, et al. Gene transfection mediated by polyethyleneiminepolyethylene glycol nanocarrier prevents cisplatin-induced spiral ganglion cell damage[J]. Neural Regen Res, 2015, 10(3): 425-431. https://doi.org/10.4103/16735374.153691

14. Liu C, Xie Y, Li X, et al. Folic Acid/Peptides Modified PLGA-PEI-PEG Polymeric Vectors as Efficient Gene Delivery Vehicles: Synthesis, Characterization and Their Biological Performance[J]. Mol Biotechnol, 2021, 63(1): 63-79. https://doi: 10.1007/s12033-020-00285-5

15. Watanabe M, Ebina M, Orson F M, et al. Hepatocyte growth factor gene transfer to alveolar septa for effective suppression of lung fibrosis[J]. Mol Ther, 2005, 12(1): 58-67. https://doi: 10.1016/j.ymthe.2005.02.019.

16. Mishra S, Webster P, Davis ME. PEGylation significantly affects cellular uptake and intracellular trafficking of non-viral gene delivery particles[J]. Eur J Cell Biol. 2004, 83(3): 97-111. https://doi: 10.1078/01719335-00363

17. Alaniz L, Cabrera $\mathrm{P}$ V, Blanco $\mathrm{G}$, et al. Interaction of CD44 with different forms of hyaluronic acid. Its role in adhesion and migration of tumor cells[J]. Cell Commun Adhes, 2002, 9(3): 117-130. https://doi.org/10.1080/15419060214522

18. Widjaja L K, Bora $\mathrm{M}$, Chan $\mathrm{P} \mathrm{N}$, et al. Hyaluronic acid-based nanocomposite hydrogels for ocular drug delivery applications[J]. J Biomed Mater Res A, 2014, 102(9): 3056-3065. https://doi.org/10.1002/jbm.a.34976

19. Cui Z, Liao J, Cheong N, et al. The Receptor for Hyaluronan-Mediated Motility (CD168) promotes inflammation and fibrosis after acute lung injury[J]. Matrix Biol, 2019, 78-79: 255271.

https://doi.org/10.1016/j.matbio.2018.08.002

20. He Y, Cheng G, Xie L, et al. Polyethyleneimine/DNA polyplexes with reduction-sensitive hyaluronic acid derivatives shielding for targeted gene
delivery[J]. Biomaterials, 2013, 34(4): 12351245. https://doi: 10.1016/j.biomaterials.2012.09.049

21. Nie L, Sun S, Sun M, et al. Synthesis of Aptamer-PEI-g-PEG Modified Gold Nanoparticles Loaded with Doxorubicin for Targeted Drug Delivery[J]. J Vis Exp, 2020(160). https://doi.org/10.3791/61139

22. Gazdhar A, Temuri A, Knudsen L, et al. Targeted gene transfer of hepatocyte growth factor to alveolar type II epithelial cells reduces lung fibrosis in rats[J]. Hum Gene Ther, 2013, 24(1): 105-116. https://doi.org/10.1089/hum.2012.098

23. Nakamura TMizuno $\mathrm{S}$ The discovery of hepatocyte growth factor (HGF) and its significance for cell biology, life sciences and clinical medicine[J]. Proc Jpn Acad Ser B Phys Biol Sci, 2010, 86(6): 588-610. https://doi.org/10.2183/pjab.86.588

24. Yaekashiwa M, Nakayama S, Ohnuma K, et al. Simultaneous or delayed administration of hepatocyte growth factor equally represses the fibrotic changes in murine lung injury induced by bleomycin. A morphologic study[J]. Am J Respir Crit Care Med, 1997, 156(6): 19371944.

https://doi.org/10.1164/ajrccm.156.6.9611057

25. Sanada F, Fujikawa T, Shibata K, et al. Therapeutic Angiogenesis Using HGF Plasmid[J]. Ann Vasc Dis, 2020, 13(2): 109115. https://doi.org/10.3400/avd.ra.20-00035

26. Mairhofer JGrabherr R Rational vector design for efficient non-viral gene delivery: challenges facing the use of plasmid DNA[J]. Mol Biotechnol, 2008, 39(2): 97-104. https://doi.org/10.1007/s12033-008-9046-7

27. Bobo D, Robinson $\mathrm{K} \mathrm{J}$, Islam $\mathrm{J}$, et al. Nanoparticle-Based Medicines: A Review of FDA-Approved Materials and Clinical Trials to Date[J]. Pharm Res, 2016, 33(10): 23732387. https://doi.org/10.1007/s11095-0161958-5

28. Liu J, Shao H, Fang S, et al. Evaluation of pharmacokinetics and pharmaco-dynamics of sinomenine-hyaluronic acid conjugate after intra-articular administration for osteoarthritis treatment[J]. Drug Des Devel Ther, 2019, 13: 657-665. https://doi: 10.2147/DDDT.S186558.

29. Thorley A J, Ruenraroengsak P, Potter T E, et al. Critical determinants of uptake and translocation of nanoparticles by the human 
pulmonary alveolar epithelium[J]. ACS Nano, 2014, 8(11): $11778-11789$. https://doi.org/10.1021/nn505399e

30. Arvizo R R, Miranda O R, Thompson M A, et al. Effect of nanoparticle surface charge at the plasma membrane and beyond[J]. Nano Lett, 2010, 10(7): 2543-2548. https://doi: $10.1021 / \mathrm{nl} 101140 \mathrm{t}$

31. Osman N M, Sexton D, WSaleem I Y. Toxicological assessment of nanoparticle interactions with the pulmonary system[J]. Nanotoxicology, 2020, 14(1): 21-58. https://doi: 10.1080/17435390.2019.1661043

32. Yeh Y C, Saha K, Yan B, et al. The role of ligand coordination on the cytotoxicity of cationic quantum dots in HeLa cells[J]. Nanoscale, 2013, 5(24): 12140-12143. https://doi.org/10.1039/c3nr04037b

33. $\mathrm{Li} \mathrm{C}, \mathrm{Lu} \mathrm{Y}, \mathrm{Du} \mathrm{S}$, et al. Dioscin Exerts Protective Effects Against Crystalline Silicainduced Pulmonary Fibrosis in Mice[J]. Theranostics, 2017, 7(17): 4255-4275. https://doi.org/10.7150/thno.20270

34. Dong J, Ma Q. Myofibroblasts and lung fibrosis induced by carbon nanotube exposure[J]. Part Fibre Toxicol, 2016, 13(1): 60. https://doi: 10.1186/s12989-016-0172-2

35. Wilson M S, Wynn T A. Pulmonary fibrosis: pathogenesis, etiology and regulation[J]. Mucosal Immunol, 2009, 2(2): 103-121. https://doi: $10.1038 / \mathrm{mi} .2008 .85$

36. Wynn T A, Ramalingam T R. Mechanisms of fibrosis: therapeutic translation for fibrotic disease[J]. Nat Med, 2012, 18(7): 1028-1040. https://doi.org/10.1038/nm.2807

37. Schleiff P L, Mazurek J M, Reilly M J, et al. Surveillance for Silicosis - Michigan and New Jersey, 2003-2011[J]. MMWR Morb Mortal Wkly Rep, 2016, 63(55): 73-78. https://doi.org/10.15585/mmwr.mm6355a7

38. Ferrante P. Asbestosis and silicosis hospitalizations in Italy (2001-2015): results from the National Hospital Discharge Registry[J]. Eur J Public Health, 2019, 29(5): 876-882. https://doi: 10.1093/eurpub/ckz003 